\title{
Response latencies of rats during behavioral thermoregulation*
}

\author{
DONALD M. SCOTT $†$ and VADEN BLACKWOOD \\ Wofford College, Spartanburg, South Carolina 29301
}

\begin{abstract}
The cutaneous thermal stimulation that elicits behavioral thermoregulatory behavior was investigated in these experiments. In Experiment I, rats were placed in a cool environment and allowed to barpress for 3-sec bursts of radiant heat reinforcement. In various phases of the study, rats could earn different intensities of anterior, posterior, or whole-body radiation. Identical response rates were exhibited at each intensity for all exposure conditions; this information, when considered with existing literature on behavioral and neurophysiological studies, suggests that the rat's thermoregulatory behavior depends on information carried by nonmyelinated fibers that supply thermoreceptors in the skin. Experiment II investigated the hypothesis that cooling of the skin is the stimulus that elicits each thermoregulatory response. Time series measures of skin temperature fluctuations and reaction times (RTs) were obtained. Tails of the RT distributions were shown to conform to exponential probability density functions, and mean RT varied linearly over the domain of reinforcement intensities used. A computer simulation model that describes temperature gradients across layers of skin was employed to estimate temperature fluctuations at the level of the cool receptors. Comparison of simulated skin temperatures with obtained RTs suggests that momentary thermoregulatory behavior is controlled mainly by cooling skin temperature.
\end{abstract}

The cue that controls responses in behavioral thermoregulation is still unknown, although many aspects of the ability of animals to maintain normal body temperature in cold environments have been studied (Carlisle, 1970; Corbit, 1970). It is well known that a fall in skin temperature is necessary for initiation of behavioral thermoregulation (Weiss \& Laties, 1961; Carlisle, 1966, 1968), but a question remains concerning the stimulus utilized by the animal when each operant response is emitted. Several possibilities exist: the animal can work (appetitively) for heat or it can work to avoid or escape cold.

A discussion by Carlisle (1970) concluded that heat intake occurs at a constant rate as a function of reinforcer duration and intensity when the former parameter is under control of the animal. He was not able to specify the sensory function that controlled the behavior, but could only assume that some temperature-dependent signal arising from on or within the animal was involved. A model was presented by Corbit (1970) intended to account for available data on thermoregulation:

$$
\text { Response }=a\left(T_{h y_{o}}-T_{h y}\right)+a b\left(T_{s_{o}}-T_{s}\right) .
$$

In this model, $\mathrm{T}_{\mathbf{h y}_{\mathrm{o}}}$ and $\mathrm{T}_{\mathbf{s}_{\mathrm{o}}}$ are temperatures of the hypothalamus and skin, respectively, that fail to elicit thermoregulatory behavior and may be considered as the core temperature and skin temperature of the rat measured at $20^{\circ}-25^{\circ} \mathrm{C}$. They are relatively constant throughout the experiment, so that behavior is mainly

*This research was supported by a grant from the Wofford College Faculty Research Program and in part by COSIP Grant GY 7666 from the National Science Foundation.

$\dagger$ The advice and support of $R$. Duncan Luce in various phases of this experiment is gratefully acknowledged; also the Wofford College Computer Center for making available their facilities. dependent on momentary hypothalamic and skin temperatures.

During behavioral thermoregulation, the sequence of response-reinforcement pairs causes $T_{s}$ to fluctuate more rapidly, and throughout a greater range, than variations in $T_{s_{o}}$. Thus, $T_{s}$ is more likely to serve as a controlling stimulus for each behavioral response than $T_{s_{o}}$ is, but the dependence of the responses on $T_{s}$ has not yet been demonstrated.

The present experiments were designed to investigate the cue utilized by rats for responding with a leverpress to obtain radiant heat reinforcement in a cool environment. The experiments were analyzed as time series rather than fixed-duration events, thereby allowing comparisons to be made between cutaneous temperatures and response events.

\section{EXPERIMENT I}

The weight of available evidence indicates that the posterior furry skin of subprimate mammals may be insensitive to mild thermal stimulation. No psychophysical experiments have been reported that have measured behavioral response thresholds of rats to small temperature changes of their skin. Studies by Kenshalo (1964) and Kenshalo, Duncan, and Weymarck (1967) employed temperature changes of the shaved skin of the thigh, back, and footpad of cats as a cue to shock avoidance behavior, and were unable to elicit the response with mild (less than $9^{\circ} \mathrm{C}$ ) warm or cool stimuli applied to skin adapted within the neutral range of $29^{\circ}-38^{\circ} \mathrm{C}$. However, Kenshalo, Duncan, and Weymarck (1967) obtained a response threshold of $1^{\circ} \mathrm{C}$ skin temperature change from cats exposed to radiant heating of a $4-\mathrm{cm}^{2}$ area of the nose adapted to $36.5^{\circ} \mathrm{C}$. More recently, Brearly and Kenshalo (1970) measured (behavioral) response thresholds in the cat to thermal stimuli applied to the upper lip by a contact stimulator 
that could also adapt the skin to temperatures between $27^{\circ} \mathrm{C}$ and $41^{\circ} \mathrm{C}$. Shock avoidance responses were elicited by stimuli only $1{ }^{\circ} \mathrm{C}$ (for warm) and $0.5^{\circ} \mathrm{C}$ (for cool) greater than required to produce reports of threshold sensation in humans after stimulation of forearm skin adapted to the same range of temperatures.

Electrophysiological studies of nerves innervating glabrous and hairy skin of subprimates generally agree that thermoreceptors in hairy skin (other than that surrounding the nose and mouth) are supplied by nonmyelinated fibers, while glabrous and oral-nasal area skin is innervated by both myelinated and nonmyelinated fibers. Thermoreceptors are divided into two classes, "warm" and "cool," and are defined neurophysiologically by the following set of criteria applied to the behavior of the afferent nerves: (1) they discharge at constant rate to constant temperature (static sensitivity), (2) they exhibit a transient change in discharge rate following sudden temperature change, such that the time derivative of the response rate is positive for warm fibers and negative for cool fibers (dynamic sensitivity), and (3) they are relatively insensitive to mechanical stimulation (selective sensitivity) (Hensel, 1970).

Furry skin of the rat, cat, and dog leg was found by Iriuchijima and Zotterman (1960) to contain both warm and cool thermoreceptors that were supplied by nonmyelinated fibers in the saphenous nerve. Another study of the saphenous nerve of the cat by Hensel, Iggo, and Witt (1960) also found nonmyelinated fibers supplying both warm and cool receptors. A microelectrode study by Stolwijk and Wexler (1971) yielded records of discharge from multifiber fascicles of the intact saphenous nerve of the cat. They used radiant heat warming of the skin and reported that "substantial warm fiber activity can be elicited from the hind limb of the cat," and that a "good deal" of that nerve activity occurs in C-fibers (nonmyelinated afferents).

Most often, myelinated thermoreceptors have been found for subprimates in glabrous skin or in the lip and nose area. In the rat, Bowman (1958) found myelinated cold fibers in the infraorbital nerve. In the cat, cold thermoreceptor afferents have been located in the lingual nerve (Zotterman, 1936; Hensel \& Zotterman, 1951 ; Dodt \& Zotterman, 1952a), the intraorbital nerve (Hensel, 1952), and in the infraorbital nerve of the dog (Iggo, 1969). Myelinated warm receptors have been found in the tongue of the cat and dog (Zotterman, 1936; Hensel \& Zotterman, 1951; Dodt \& Zotterman, $1952 \mathrm{~b})$, and in the infraorbital nerve of the cat by Hensel (1968a,b). A few nonmyelinated thermoreceptors have also been found in glabrous or oral-nasal area skin: warm C-fibers were found in abundance in the scrotum of the rat by Iggo (1969); Hensel, Iggo, and Witt (1960) found cool C-fibers in the infraorbital nerve of the cat; and some warm and cool $C$-fibers were found in the infraorbital nerve of the cat and dog by Iriuchijima and Zotterman (1960).
To summarize the relevant findings, the cat could not be conditioned to respond with shock avoidance to mild temperature changes of his (furry) leg or back. The animal could be conditioned to respond to mild thermal stimuli of the nose and lip, however. Thermoreceptors in the skin of the thigh and back seem to be innervated by nonmyelinated fibers, whereas those of glabrous skin and oral-nasal face skin seem to be supplied by both myelinated and nonmyelinated fibers. No behavioral studies are available on the rat, but the furry and glabrous/oral-nasal regions of that animal's skin seem to be innervated much like the cat and dog skin.

Behavioral thermoregulation is assumed to depend on cutaneous thermosensitivity, and the ability of the rat to maintain his body temperature by behavioral means might be expected to vary as sensitivity of the skin to temperature stimulation varies. If the nonmyelinated afferent fibers that innervate thermoreceptors can be used by the rat to regulate leverpressing for radiant heat reinforcement, then the behavior ought to be as accurately controlled whether reinforcement is confined to the posterior (furry) skin or the anterior (face) skin. However, if the animal cannot use thermosensory input from the $\mathrm{C}$-fibers of his furry skin regions, then thermal regulation would be expected to vary, depending on whether the anterior (facial) or posterior skin receives heat reinforcement.

\section{Procedure}

The Ss employed in this study were two adult albino rats of mixed stock, one male (A nimal I) and one female (Animal II).

They were maintained for the experiment at $90 \%$ of their free-feeding weights. They were individually caged, fed Purina Chow, and had free access to water throughout the study. Each animal was clipped of all body hair prior to each experimental session.

The test chamber was a modified soft-drink cooler that maintained an ambient forced-air temperature of $2^{\circ} \mathrm{C}$. The animals were enclosed in a wire cage, $6 \times 7 \times 19 \mathrm{~cm}$, made of $1 / 2$-in. hardware cloth that contained at one end a lever-activated microswitch for control of the heat lamp circuit. Two 375-W heat lamps were positioned $12 \mathrm{~cm}$ above the cage, and the lamp output was filtered by Corning No. 2540 infrared transmitting filters. Solid state timing and control circuits were located in an adjoining room. When the lever was depressed, the heat lamps were turned on for $3 \mathrm{sec}$; responses occurring while the lamps were on had no effect on the lamp timing.

Lamp intensities of $510,390,276,227,186$, and $153 \mathrm{~W}$ were presented in a randomized sequence. For training purposes, the animals were run in daily sessions for a total of four times under each lamp setting. Each session lasted 5-6 h. At the end of this training procedure, data was gathered from a new random sequence of 5-6-h sessions. Each of the six intensities occurred four times in the new sequence, and each session was long enough for at least 500 reinforcements to occur.

For the anterior- and posterior-radiation conditions, a mask was inserted between the lamp and the animal so that, under the anterior condition, radiant heat was received by the skin forward of a transverse imaginary line located just behind the front legs. In the posterior-heat condition, the mask was reversed and the heating was confined to the rear portion of the rat (behind the imaginary line). Although skin temperature was not measured to confirm that radiant heat was confined to unshaded portions of 
the animal, the skin on the shaded areas of the rat always felt cool to the touch when the animal was removed from the chamber, whereas the unshaded skin was warm. Ambient temperatures measured with a thermometer suspended inside the chamber varied less than $2^{\circ} \mathrm{C}$ (within the range of $2^{\circ}-4^{\circ} \mathrm{C}$ ) during experimental sessions, for anterior, posterior, or whole-body radiation conditions. The data collection procedures for the anterior- and posterior-heat conditions were identical to those used in the whole-body-heat condition.

\section{Results}

Mean reinforcements per minute were calculated for each animal for the various stimulus conditions. There was essentially no difference among reinforcement rates due to radiation of the whole body vs anterior vs posterior portions of the animal. Lines were fitted by the method of least squares to the points that related stimulus magnitude (in watts) with the mean reinforcement rates for whole-body, anterior, and posterior conditions, respectively. These data and the fitted lines are presented in Fig. 1 for both animals. The data are summarized by comparing response rates for some stimulus value and the associated line slopes (see Table 1). Reference to the data in Table 1 shows that the mean reinforcement rates (per minute) at 153-W stimulus intensity are within the range of 7.91-8.26 for Animal I and 6.68-7.65 for Animal II; slopes for
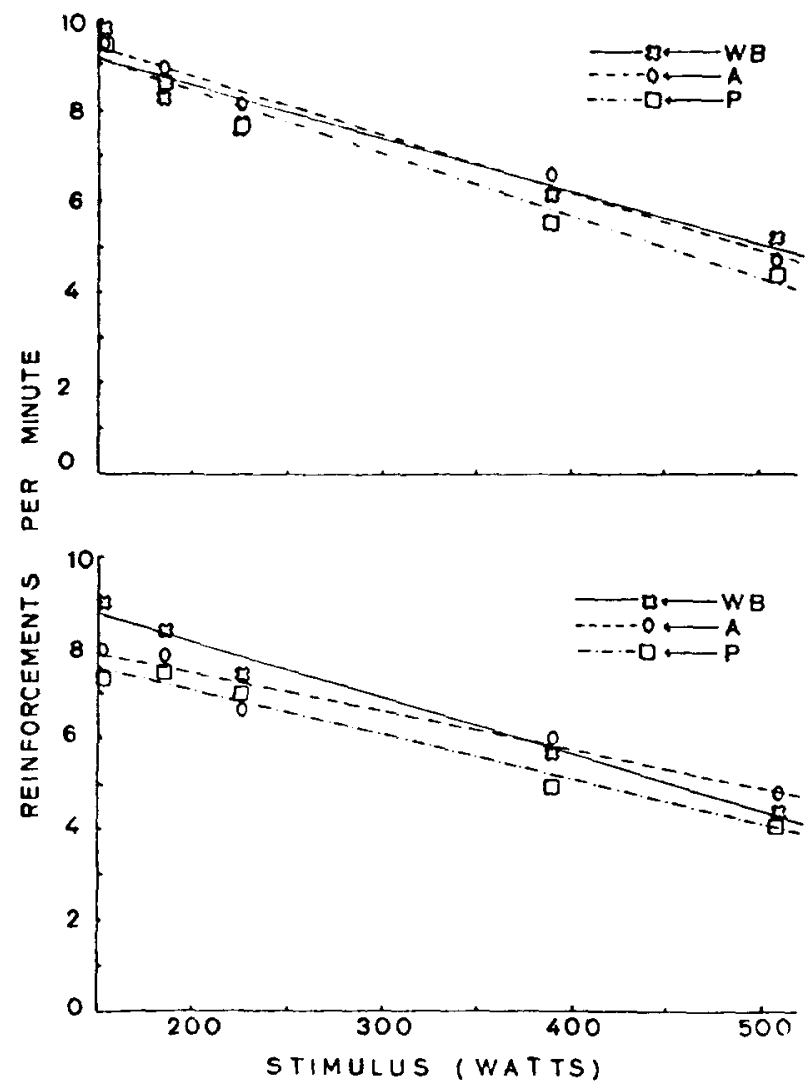

Fig. 1. Mean reinforcement rates for whole-body (WB), anterior $(A)$, and posterior $(P)$ radiation at each reinforcement intensity. Top, A nimal I: bottom. Animal II.
Table 1

Mean Reinforcement Rates at 153-W Stimulus Intensity and Slopes of Best-Fit Regression Lines for Experiment I

\begin{tabular}{lcccc}
\hline \multirow{2}{*}{ Heat } & \multicolumn{2}{c}{ Rat I } & \multicolumn{2}{c}{ Rat II } \\
Condition & Mean & Slope & Mean & Slope \\
\hline Whole Body & 8.11 & -.012 & 7.65 & -.012 \\
Anterior & 8.26 & -.013 & 7.10 & -.008 \\
Posterior & 7.91 & -.014 & 6.68 & -.010 \\
\hline
\end{tabular}

Animal I range from -.012 to -.014 and from -.008 to -.012 for Animal II. There is no apparent pattern in the ordering of slopes or response rates.

\section{Discussion}

The results of this study have shown that mean heat reinforcement rates for rats in a cold environment do not depend systematically on whether the animals are exposed to whole-body radiation or whether they are reinforced by heating either the anterior or posterior half of their bodies only. The positive result of the present experiment is the indication that rats may be able to use nonmyelinated fiber information in the execution of an operant behavior, in view of the inability of electrophysiological studies to reveal myelinated fibers innervating thermoreceptors in the caudal furry skin of the subprimate mammals rat, cat, or dog. Although thermoreceptors have been identified in the scrotum of the rat, it seems unlikely that the thermal sensitivity of the scrotum could account for the data of this experiment, because (1) the scrotum is in a shielded position relative to the radiant source located directly above the animals, and (2) quite similar data were obtained from the male and female Ss. Thus, it is concluded that the rat can use temperature information from the entire dorsal surface of his body when behaviorally regulating its body temperature, and the next experiment investigated the hypothesis that cooling of the skin, rather than warming, is the cue that controls responding for fixed-duration radiant heat reinforcement in a cold environment.

\section{EXPERIMENT II}

Measurements of subcutaneous and hypothalamic temperatures during behavioral thermoregulation have revealed that a fall of skin temperature and central temperature usually precede initiation of responding for heat in naive rats, but that experienced Ss usually do not wait for a drop in core temperature before commencing to work (Weiss \& Laties. 1961; Carlisle, 1968). No experiment has identified the sensory cue that controls each response for heat once the animal has begun behavioral thermoregulation.

One way to investigate the dependence of the thermoregulatory response on momentary skin temperature is to regard the thermoregulation experiment as a time series event. rather than as a fixed 


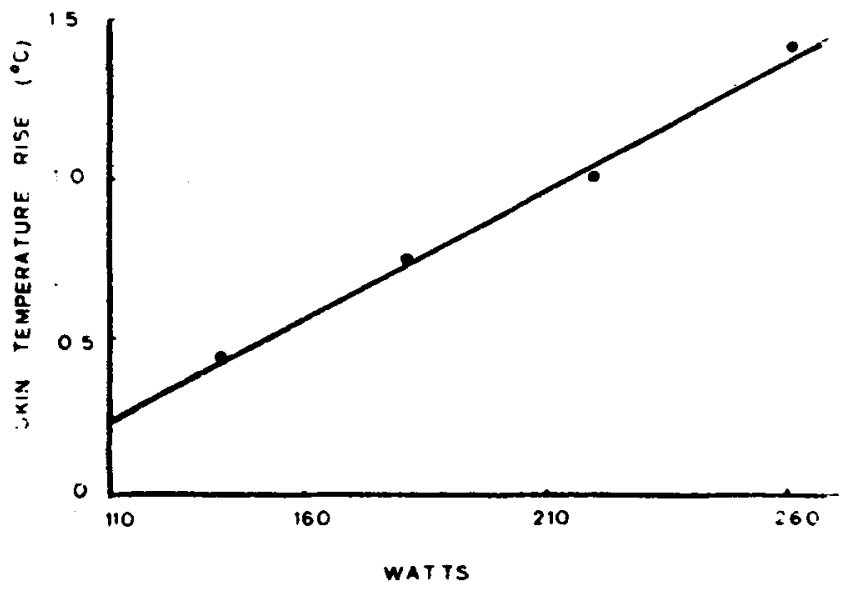

Fig. 2. Skin temperature rise produced by each reinforcement intensity. Exposure duration was 3 sec.

duration experiment, and to compare response latencies to measured topography of skin heating and cooling. In Experiment II, conditional probability of response was calculated as a function of time after reinforcement and compared to skin temperature decay curves, in order to relate responding to skin temperature.

\section{Procedure}

Two groups of rats were used: A, containing two rats, and B, containing four rats. They were maintained identically to those of the first experiment. Radiant heat reinforcement for this experiment was obtained from two $250-W$ heat lamps rather than from 375-W lamps as in Experiment I. Otherwise, the test chamber and control equipment were the same, with the additional feature that latencies were measured from the end of the reinforcement pulse until the first subsequent response (which, of course, initiated the next reinforcement), and automatically punched into paper tape for analysis on the Wofford College time-sharing computer terminal.

The animals in Group A were trained at six lamp intensities $(399,301,251,210,171,133 \mathrm{~W})$, as in Experiment I. For data collection, a new random sequence of lamp intensities was obtained, in which each of the six intensities occurred four times. The animals were run in daily sessions according to this new sequence until at least 500 reinforcements and the associated responses had been recorded from each session.

Group B animals were trained for a minimum of $10 \mathrm{~h}$ at each of four intensities $(251,210,171,133 \mathrm{~W})$. Then at least 2,000 reaction-time responses for each stimulus magnitude were punched into computer tape. Responses were obtained from two or three sessions run on successive days under the given stimulus condition. After a complete set of data was collected for an animal at one stimulus magnitude, the animal was shifted to another magnitude and given an additional $10-15 \mathrm{~h}$ practice at the new reinforcement magnitude before data was collected under that condition

Skin temperature for the animals in Group B was measured with a VECO NM-29-10-324 needle thermistor inserted into the dorsal skin of a lightly anesthetized (Nembutal) rat. The thermistor was connected via a bridge circuit to a Gilson polygraph chopper amplifier, and temperature changes were drawn on moving chart paper. Skin temperature changes per various lamp intensities (ranging from 133 to $251 \mathrm{~W}$ ) were plotted, and the thermistor was calibrated in a water bath using a mercury thermometer. Skin temperature rise was linearly related to lamp intensity. from $0.44^{\circ} \mathrm{C}$ at $133 \mathrm{~W}$ to $1.40^{\circ} \mathrm{C}$ at $251 \mathrm{~W}$ (see Fig. 2).

\section{Results and Discussion}

Polygraph tracings of the skin temperature warming and cooling curves are shown in Fig. 3. The smallest lamp intensity $(133 \mathrm{~W})$ warmed the skin by $.44^{\circ} \mathrm{C}$ during the 3 -sec stimulus, and an additional $3 \mathrm{sec}$ were required for cooling to near the original temperature. Warmer stimuli of $171 \mathrm{~W}$ warmed the skin by $0.74^{\circ} \mathrm{C}$, which cooled in about $5 \mathrm{sec} ; 210 \mathrm{~W}$ heated the skin $1.0^{\circ} \mathrm{C}$, and about $6 \mathrm{sec}$ were required for cooling. The warmest (251 W) lamp intensity warmed the skin by $1.4^{\circ} \mathrm{C}$ during the 3-sec stimulation, and nearly $9 \mathrm{sec}$ were necessary for cooling to the original temperature.

Momentary skin temperatures were measured from the cooling curves of the polygraph tracings, and exponential decay curves were fitted to the data by the least-squares method. Skin temperature decay curves are presented in Fig. 4. From these curves, the time constant $(\tau)$ of the cooling curve was calculated as the time required for the skin temperature, $T$, to cool to $1 / \mathrm{e} \times T_{0}$, where $T_{0}$ is the skin temperature at the beginning of the reinforcement heat burst. A plot of the time constants as a function of stimulus magnitude is presented in Fig. 5. The measures of $\tau$ can be regarded as measures of the rates of cooling of the skin after the various stimulus magnitudes were presented. The nonlinearity apparent at stimulus magnitude of $133 \mathrm{~W}$ may be due to a change in heat conductivity of the skin following heating to $0.44^{\circ} \mathrm{C}$ by that lamp intensity as

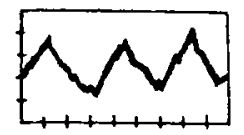

$133 \mathrm{~W}$

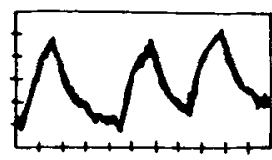

$171 W$
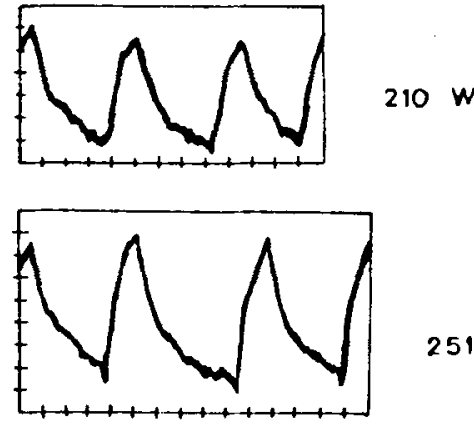

$251 W$

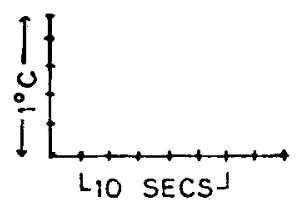

Fig. 3. Polygraph tracings of skin temperature fluctuation at each reinforcement intensity. 
compared to the warming that followed reinforcements of 171,210 , and $251 \mathrm{~W}$. Evidence has been marshaled by Kenshalo (1970) that venular smooth muscle may become quite reactive when epinephrine (released at time of thermal stress) is combined with a fall in skin temperature of $1^{\circ} \cdot 2^{\circ} \mathrm{C}$. Perhaps the skin warming produced by the lowest reinforcement intensity was too mild to stimulate venular smooth muscle.

Reaction times (RTs) were sorted into a frequency distribution having class width of $4 \mathrm{sec}$. Such distributions of response latencies typically are unimodal and stiongly skewed toward longer time values, and specifying the mode or mean and standard deviation does not convey much information about the time-dependent (stochastic) properties of various sets of data that are similarly distributed. Consideration of the present experiment, where heat loss is expected to progressively enhance propensity to respond during the interval between reinforcements, suggests that a statistic would be appropriate which reveals the changing likelihood of response as a function of time since reinforcement. Such a statistic is the conditional density function (McGill. 1963) (or "hazard function") $h(t)$. which calculates the momentary probability $p=h\left(t_{i}\right)$ of response conditional on no response occurring at time

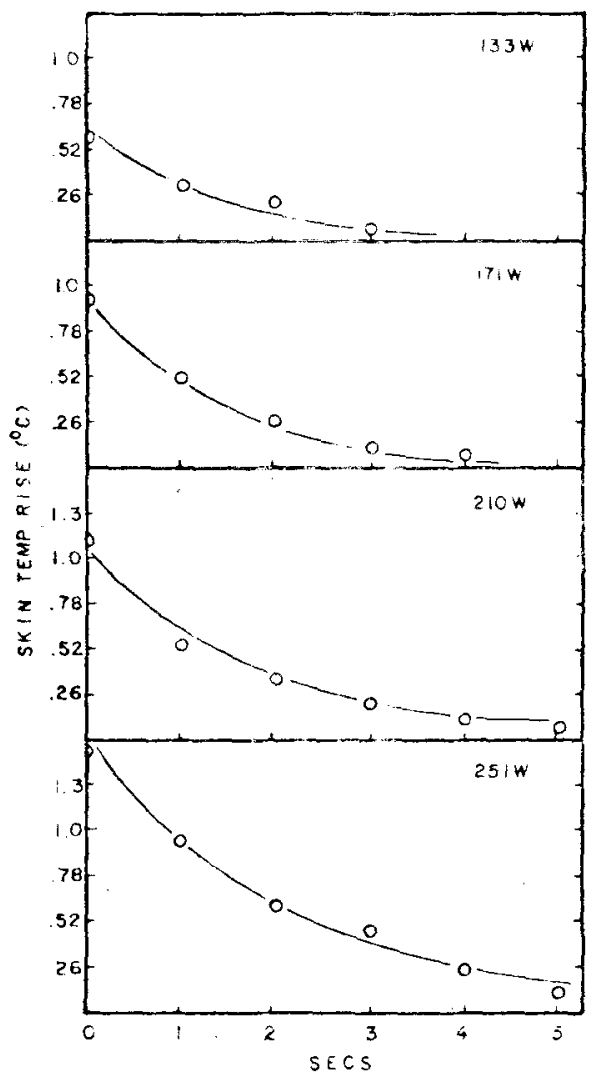

Fig. 4. Skin temperatures during the cooling phase of the response-reinforcement cycle, and best-fit exponential decay curves for various reinforcement intensities.

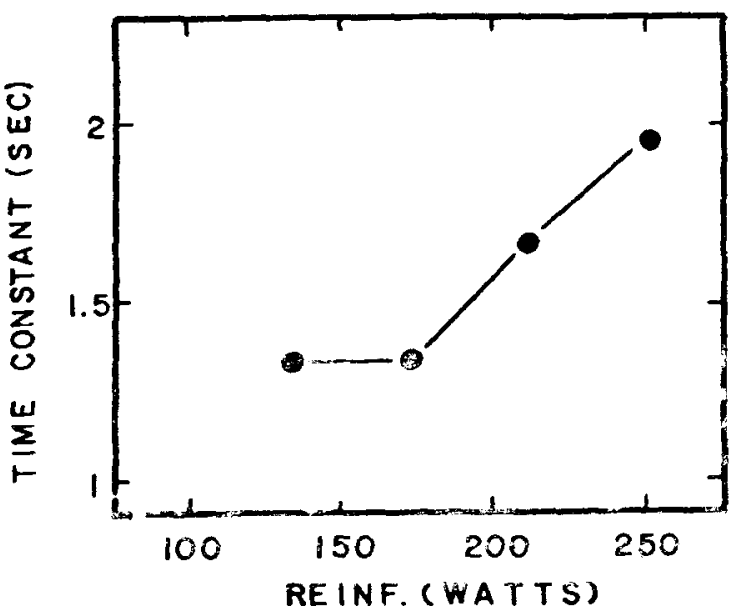

Fig. 5. Time constants for the exponential decay curves of Fig 4.

$t<t_{i}$. For the data of this experiment, which are sorted into discrete intervals,

$$
h\left(t_{i}\right)=\frac{\text { frequency in interval } t_{i}}{\sum_{j=i}^{N} \text { frequency in interval } t_{j}}
$$

where $\mathrm{i}=1,2, \cdots, \mathrm{N}$ classes. Hazard function values can be interpreted as being measures of instantaneous response rates.

Figure 6 displays a typical frequency distribution for Rat $\mathrm{V}$ of Group $\mathrm{A}$ and the associated $\mathrm{h}(\mathrm{t})$ values. Figures $7 \mathrm{a}$ and $7 \mathrm{~b}$ display $\mathrm{h}(\mathrm{t})$ for all six stimulus magnitudes, for both animals of Group A. In each curve, only $h(t)$ values representing 20 or more $R T$ s are plotted, so that probabilities can be estimated with a resolution of \pm .025 . The conditional probabilities of responding in Figs. $7 \mathrm{a}$ and $7 \mathrm{~b}$ decrease with time, rather than increase, for most of the stimulus conditions. Furthermore, with the smallest reinforcement magnitude, the likelihood of responding drops more rapidly than for any other reinforcement magnitude, declining from .75 to .25 within $16 \mathrm{sec}$. Clearly, the hazard function statistic describes an animal that is less and less likely to respond for reinforcement as time goes by and skin temperature cools, a seemingly paradoxical result. One would expect stimulation of cool receptors that would serve as a cue for the next response to be more and more likely to occur as the skin cools steadily between reinforcements. Since the present analysis does not reveal a probability of response that increases with time between reinforcements, either the analysis must be modified or it must be concluded that skin cooling does not (directly) control responses for heat reinforcement.

The latter hypothesis seems implausible, so a modified procedure for data analysis was employed using data from a new group of animals (Group B). Examination of the animal's behavior in the apparatus revealed that 


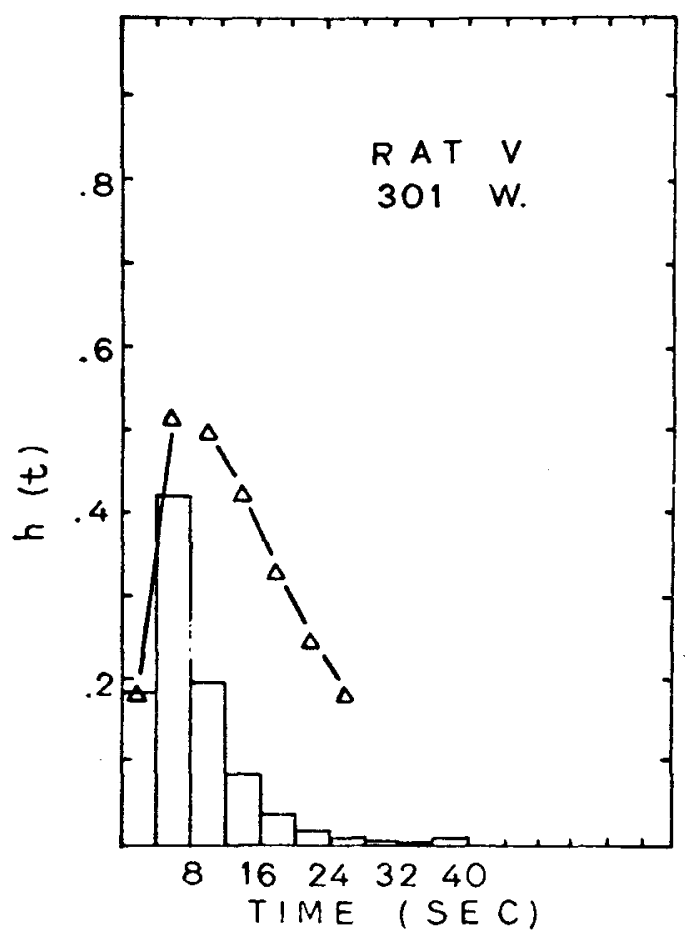

Fig. 6. Frequency distribution of RTs into 4-sec-wide bins for $R$ at $V$ at $310-W$ reinforcement intensity. Triangles are hazard-function values, $h(t)$, for each $R T$.

behaviors other than simple reaction-time responses occurred. Occasionally, the animal would huddle, sniff, scratch, etc., and in doing so his response was spuriously lengthened beyond a simple reaction-time value. The solution to this problem was to eliminate long RTs from statistical consideration, and in the absence of a compelling theoretical or experimental criterion for maximum reaction time, the upper limit of $18.7 \mathrm{sec}$ was established. The advantage of defining a maximum RT for the data is that latencies dependent on behavior other than simple reaction times are separated out from the RTs; thus, the remaining RTs can be shown to constitute a random variable in the behavioral repertoire of the animal. For the data of this experiment, an $18.7 \mathrm{sec}$ maximum RT was found (empirically) to isolate the RTs that behave as a random variable.

At least 2,000 RTs were collected from each of the four animals in Group B at each of four $(251,210,171$, and $133 \mathrm{~W}$ ) reinforcer magnitudes. The RTs were sorted into half-second-wide bins, and only those response times were used which fell in the right-hand tail of the distribution from the modal class to $18.7 \mathrm{sec}$, inclusively. The reason for considering only the tail of the RT distribution in this data analysis is derived from a paper by Luce and Green (1972) in which they presented evidence in support of a two-stage model for simple reaction-time mechanisms to sensory stimuli. The model supposes a decision process that detects changes in afferent neural traffic and occasionally initiates effector activity in a response-generating mechanism. It was assumed that the response processes behaved as bounded (at $1 / 2 \mathrm{sec}$ ) random variables, and that the behavior of the decision process was described by an unbounded variable. Evidence was presented by them to support the boundedness assumption for the response mechanism, thus allowing the tail of the reaction-time distribution to be separated from the bounded, shorter response process latencies and studied in isolation. In the present experiment, where the tail was defined on the time interval beginning with the modal class and extending to $18.7 \mathrm{sec}$, the first $1.5 \mathrm{sec}$ were excluded from the data of most distributions, except the following: at $251 \mathrm{~W}$, for Animals 2 and V2, $2 \mathrm{sec}$ excluded; at $210 \mathrm{~W}$, for Animals $T$ and $V 2,2.5 \mathrm{sec}$ excluded; at $210 \mathrm{~W}$, for Animal SW, $2 \mathrm{sec}$ excluded. It was necessary to exclude up to $2.5 \mathrm{sec}$ from the left-hand tail of these data in order to isolate the RTs falling in the modal class and up to $18.7 \mathrm{sec}$. A possible reason for the necessity for excluding left-tail RTs that were up to five times as long as the longest ones excluded by Luce \& Green (1972) can be obtained from the skin cooling curves of Fig. 3. The skin cools about $.4^{\circ} \cdot .5^{\circ} \mathrm{C}$ during the first $2 \mathrm{sec}$ following the reinforcement, for all heat intensities. Thus, it is plausible that during the time when the RTs were measured, after termination of the reinforcer, the skin had to cool by some threshold amount before sufficient skin cooling could occur to serve as a cue for the response.

The tails of the RT distributions were tested for

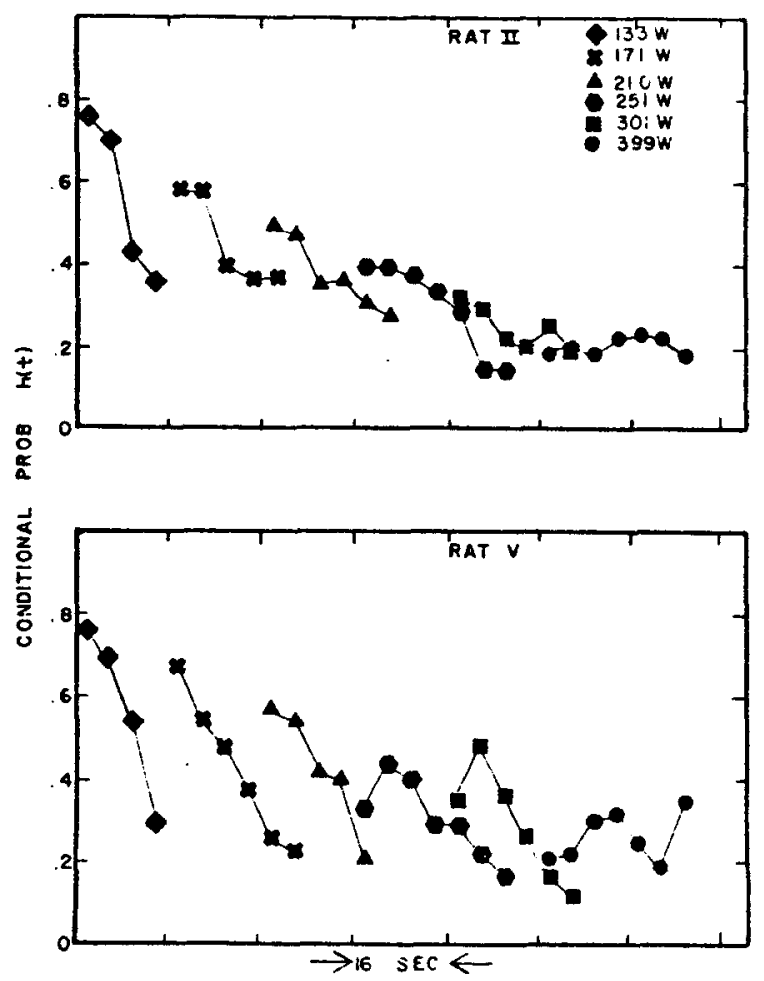

Fig. 7. (a and b) Hazard functions for Animals II and $\mathrm{V}$ at various reinforcement intensities. 
goodness-of-fit to exponential probability density functions. Evidence is available (Luce \& Green, 1970) that the tail of distributed human RTs, following an auditory signal, conforms reasonably well to exponential probability density function, and it was desired to determine whether rat thermoregulatory RTs are similarly described. Consequently, the RTs in the tails of the distributions from our present experiment were fitted with exponential probability density functions [of the form $f(t)=\lambda e^{-\lambda t}$ ].

The maximum likelihood statistic for estimation of the parameter, $\lambda$, of the exponential probability density function is given as $1 / \bar{X}$, where $\bar{X}$ is the mean of the obtained response latencies. In the present experiment, however, response latencies $t_{i}\left(0 \leqslant t_{i} \leqslant T\right)$ were only measured up to some maximum time, $T(T=18.7 \mathrm{sec})$, yielding a truncated exponential density function. Thus, the density function may be written as

$$
f_{\lambda}(t)= \begin{cases}\lambda e^{-\lambda t} / 1-e^{-\lambda T} & 0 \leqslant t \leqslant T \\ 0 & \text { otherwise }\end{cases}
$$

where the numerator is the usual exponential density function, and the denominator may be thought of as the integral of the exponential density curve from 0 to $T$. Note that as $T$ grows large and approaches $\infty$, the denominator becomes unity, so that for any $0<T<\infty$ the density evaluated at any time $t_{i}$ is increased by a scalar value proportional to the part of the exponential density function included in the sample truncated at some particular time, T. The function in Eq. 2 is in fact a density, because it integrates to unity over all $0 \leqslant t_{i} \leqslant T$. Then the maximum likelihood function is

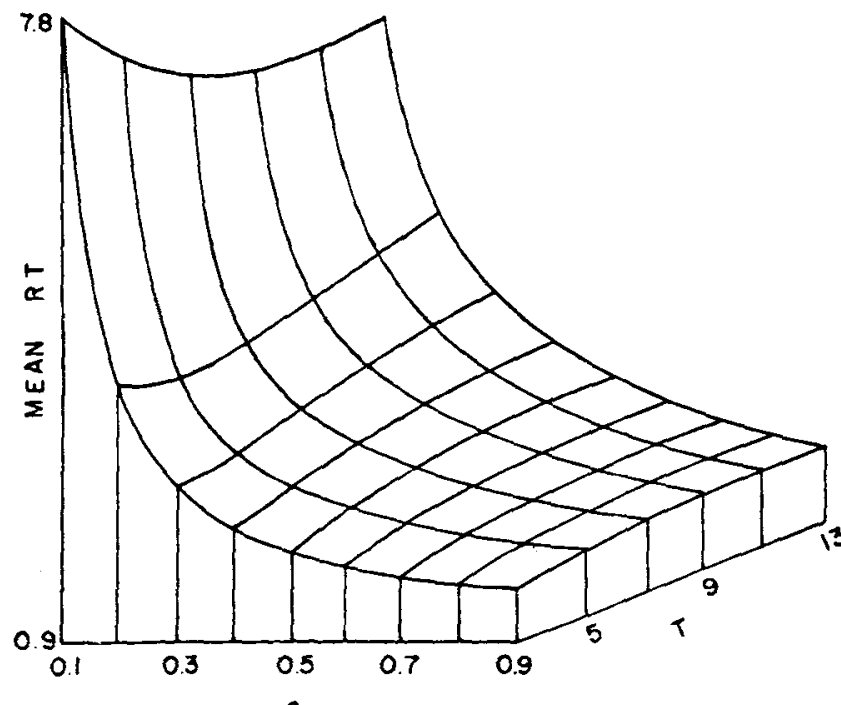

Fig. 8. Plot of the maximum likelihood statistic for the truncated exponential density function. The graph shows how the arithmetic means of data (ordinate values) depend on combinations of the exponential parameter, $\lambda$, and time, $T$, when the random process is terminated.

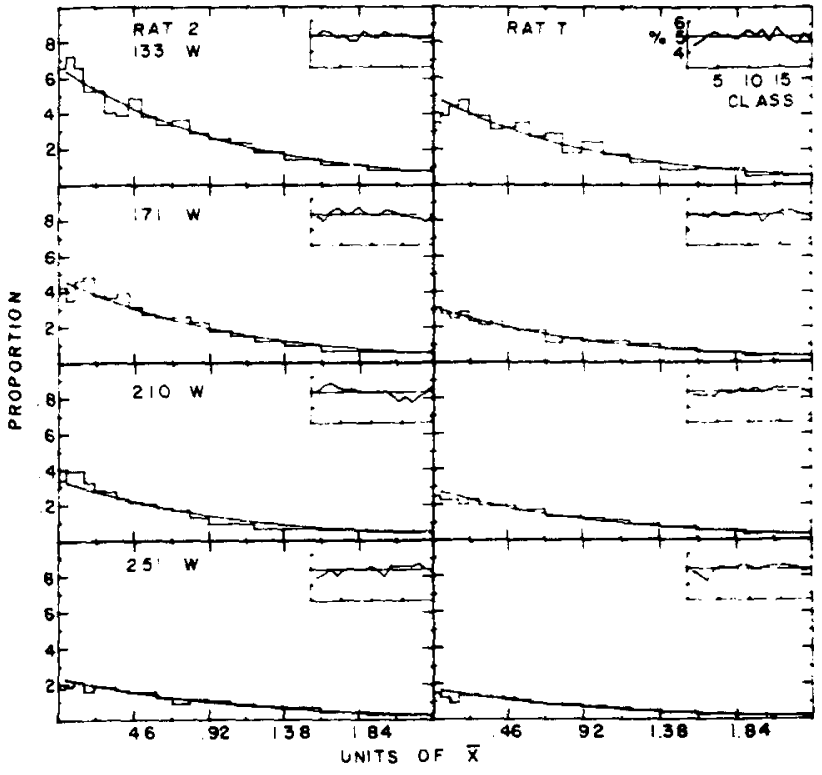

Fig. 9. RTs distributed into equal-probability intervals and fitted with exponential probability density functions, for Animals 2 and $T$.

$$
L=\prod_{i=1}^{n} f_{\lambda}\left(t_{i}\right)
$$

which yields

$$
\frac{1}{\lambda}\left(1-\lambda T e^{-\lambda T}\right)=\frac{1}{n} \sum_{i=1}^{n} t_{i}
$$

The right side is the arithmetic means of data; the left side is plotted on the ordinate of Fig. 9 as a function of $\lambda$ and $T$. Maximum likelihood estimates of $\lambda$ are obtained graphically by entering Fig. 8 from the ordinate, using the means of the obtained data and finding $\lambda$ determined by the curve associated with the appropriate T. Figures 9 and 10 present the equal-probability histograms and best-fit exponential curves for each animal under each reinforcement condition. The inset associated with each distribution presents the obtained proportion of RTs for each class $\left(t_{i}, t_{i+1}\right)$; the solid horizontal line describes the expected .05 proportions for each class if the RT distribution were perfectly exponential.

The chi-square goodness-of-fit measures for the various sets of data presented in Figs. 9 and 10 are summarized in Fig. 11. The majority of the obtained chi-square values (solid outlines) are less than 39.247, the value equal to the mean plus 2 standard deviations. Examination of the extreme values revealed that two of them (Rat SW, $171 \mathrm{~W}, \chi^{2}=74.21$; Rat V2, $171 \mathrm{~W}$, $\chi^{2}=82.85$ ) occurred because of too few obtained RTs at the shortest times, $t<.34 \mathrm{sec}$ for $S W$ and $t<.1 \mathrm{sec}$ for V2. Chi squares calculated without these small RT classes are 39.97 for SW and 38.87 for V2, much closer 


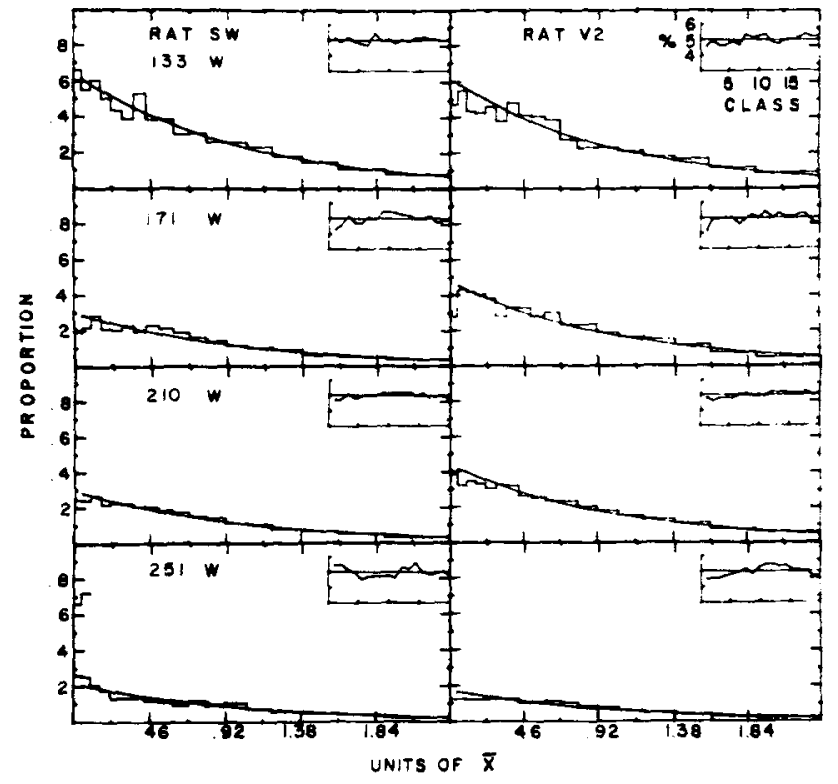

Fig. 10. RTs distributed into equal-probability intervals and fitted with exponential probability density functions, for Animals SW and V2.

to the expected range $[0, x]$, where $\mathrm{x}$ is the mean plus 2 standard deviations and are represented by dashed outlines in Fig. 11. The other two extreme chi-square values correspond to distributions that have too many RTs in the first $1.5 \mathrm{sec}$ (Rat 2, 210 W) and too few in the first $2 \mathrm{sec}($ Rat V2, $251 \mathrm{~W})$; they are simply not exponentially distributed.

The preceding analysis has shown that, given certain assumptions about short-latency (bounded at $1 / 2 \mathrm{sec}$ ) random variables and the dependence of extremely long latencies on processes other than simple reaction time, thermoregulatory behavior of the rat can be shown to behave as a random variable similar to the RTs of humans that follow auditory stimuli. Since most RTs are shown to be exponentially distributed, the mean RT can be estimated by the reciprocal of the exponential parameter, $1 / \lambda$. A plot of the mean RT vs stimulus magnitude is presented in Fig. 12. The mean RT values rise rather linearly for most animals; the mean RT for all animals, at various reinforcement magnitudes, is $1.66 \mathrm{sec}$ at $133 \mathrm{~W}, 266 \mathrm{sec}$ at $171 \mathrm{~W}, 3.00 \mathrm{sec}$ at $210 \mathrm{~W}$, and $5.08 \mathrm{sec}$ at $251 \mathrm{~W}$.

RTs that are shown to be distributed exponentially are therefore determined by the operation of a single free parameter, $\lambda$. Thus, it seems desirable to investigate whether some process can be specified within the animal that controls the behavior of the parameter. One such possibility is that the central nervous system of the rat simply reads the information incoming from the periphery and responds rather automatically when the receptor enters some specified state. Thus, it seemed useful to study the thermal behavior of the skin during the course of each reinforcement-response cycle.

A mathematical model that describes the thermal behavior of the outer $10 \mathrm{~mm}$ or so of human skin, when subjected to infrared radiation, was published by Stolwijk and Hardy (1965). The model consisted of a set of eight differential equations and was also presented as an analog computer program. A digital computer program was kindly provided by Dr. Stolwijk (personal communication) that essentially duplicated the analog version. The Stolwijk-Hardy model postulates eight layers of skin that differ in regards to their thickness, thermal conductivity, density, specific heat, and absorption coefficient. In each layer, heat is gained by absorption from the radiant source and by conduction from warmer adjacent layers; heat is lost from each layer by conduction and radiation. Functionally, the model assumes an initially linear thermal gradiant from deep tissues to the surface, then (stepwise) calculates changes in temperature for the various layers. Thus, the model would predict thermal conditions during the reinforcement (heat) time; we modified it so as to describe the behavior of the skin during the cooling phase that followed termination of the reinforcement heat.

A plot of the results of running the Stolwijk-Hardy program under one of our experimental conditions $(210 \mathrm{~W})$ is presented in Fig. 13. The program was first run to simulate warming of the skin for $3 \mathrm{sec}$. We initially assumed a linear temperature gradient from $33.5^{\circ} \mathrm{C}$ at a depth of $5.8 \mathrm{~mm}$ to $35^{\circ} \mathrm{C}$ at the surface. After $3 \mathrm{sec}$ of exposure to $50 \mathrm{mcal} / \mathrm{sec} / \mathrm{cm}^{2}$ infrared radiation, the temperature gradient was obtained that is

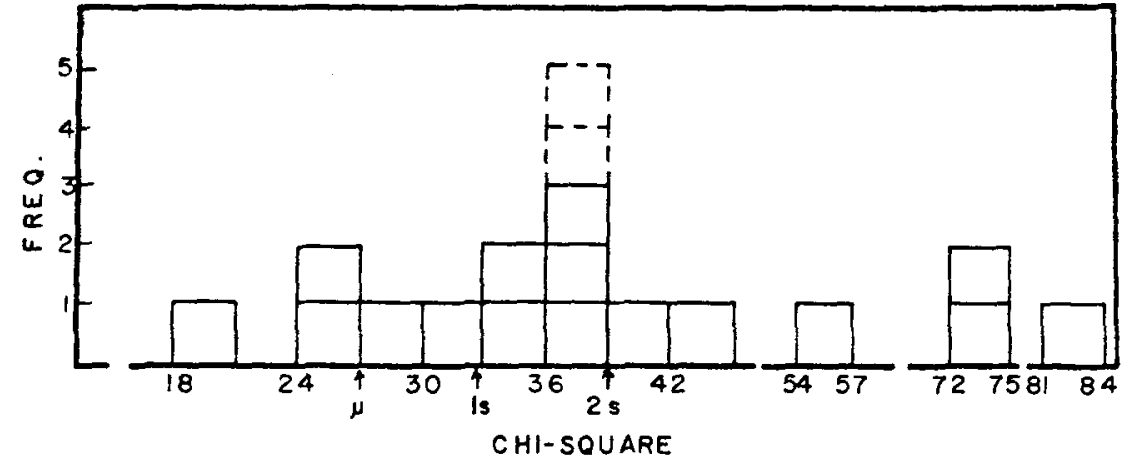

Fig. 11. Distribution of goodness-of-fit (chi-square) measures for data of Figs. 9 and 10. Dashed outlines are recalculated chi squares for two of the extreme large solid blocks when a short-duration early interval is omitted. 


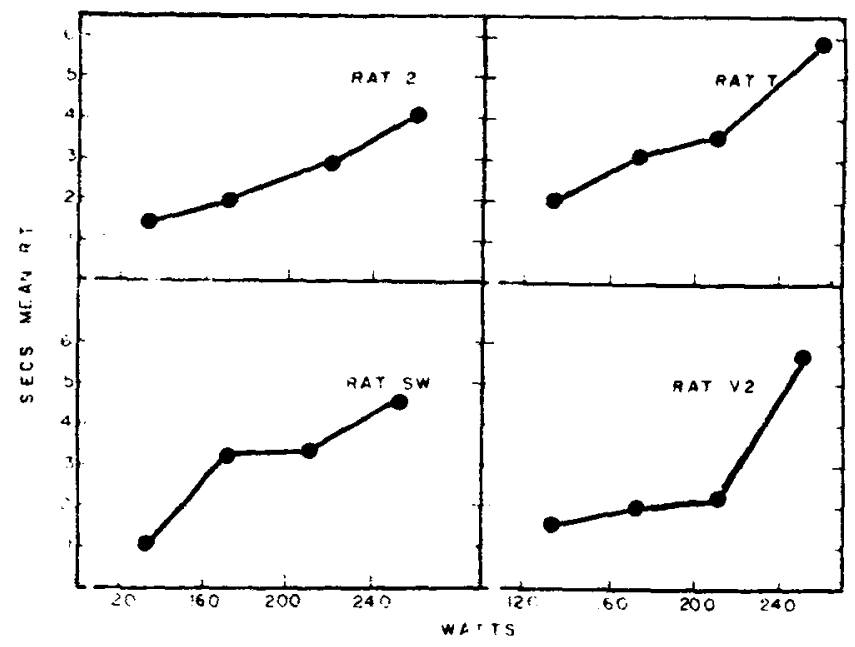

Fig. 12. Mean RTs obtained at various reinforcement intensities.

represented by the topmost of the set of curves in Fig. 13. Then the program was run to simulate cooling, and the final gradient obtained from the warming simulation was entered as the initial (time $=0 \mathrm{sec}$ ) gradient for the cooling simulation. The gradients obtained at successive 0.8-sec intervals for $4 \mathrm{sec}$ of simulated cooling in an ambient temperature of $2^{\circ} \mathrm{C}$ are shown in Fig. 13.

Before the model could be considered useful for our application, it must be shown to agree with experimentally measured data. Figure 14 presents the results of a comparison of the behavior of the model with the behavior of the animal's skin. Experimentally measured skin (surface) temperatures are represented by abscissa coordinates, and calculated temperatures (obtained from the model) are represented on the ordinate. Each square describes the comparison of experimental and theoretical data at each of the reinforcement magnitudes used in our study. The squares are for maximum warming of the skin $\left({ }^{\circ} \mathrm{C}\right.$ change in skin temperature) during the $3 . \mathrm{sec}$ reinforcement phase of the response-reinforcement cycle; the circles are for surface temperature at mean RT that followed each reinforcement magnitude. Perfect agreement betweer experimental and simulated results would appear in this plot as a linear relationship with slope of one, passing through the origin. The obtained best-fit lines fitted by the least-squares method have a slope of one (for both warming and cooling), with $\mathrm{Y}$ intercepts of -.03 (warming) and -.08 (cooling).

The results of skin cooling simulations using the Stolwijk-Hardy model describe a sequence of temperatures that decrease steadily after the cessation of heat. The exponential decay curves fitted to measured skin temperatures following reinforcement (Fig. 4) describe the time course of only the first few seconds of cooling; it is unlikely that skin temperature would asymptote at some value well above ambient temperature; rather, it would be expected to approach thermal equilibrium with the environment. The Stolwijk-Hardy model supports that expectation, thus allowing measurement of the time when $T_{\mathbf{s}}$ declines to the level that existed at the time of the previous response for heat. That preresponse temperature is taken to be directly related to the theoretical $\mathrm{T}_{\mathbf{s}_{0}}$ in this analysis.

We considered the simulation model to be useful because of the agreement between the calculated temperatures and those measured experimentally at the skin surface. The model was then used to calculate subcutaneous temperatures at a depth of $0.2 \mathrm{~mm}$ (the four th skin layer) that could not be measured directly. It has been hypothesized (Bazett, 1941) that cool receptors probably lie in the vicinity of $0.17 \mathrm{~mm}$ below the skin surface, so we investigated the hypothesis that reaction times in our thermoregulation experiment depended on stimulation of cool receptors in the skin for their occurrence. A plot of mean RT vs the time (in
Fig. 13. Temperature gradients across eight skin layers calculated from the computer simulation model of skin response to radiant heat, during the cooling phase of the response-reinforcement cycle.

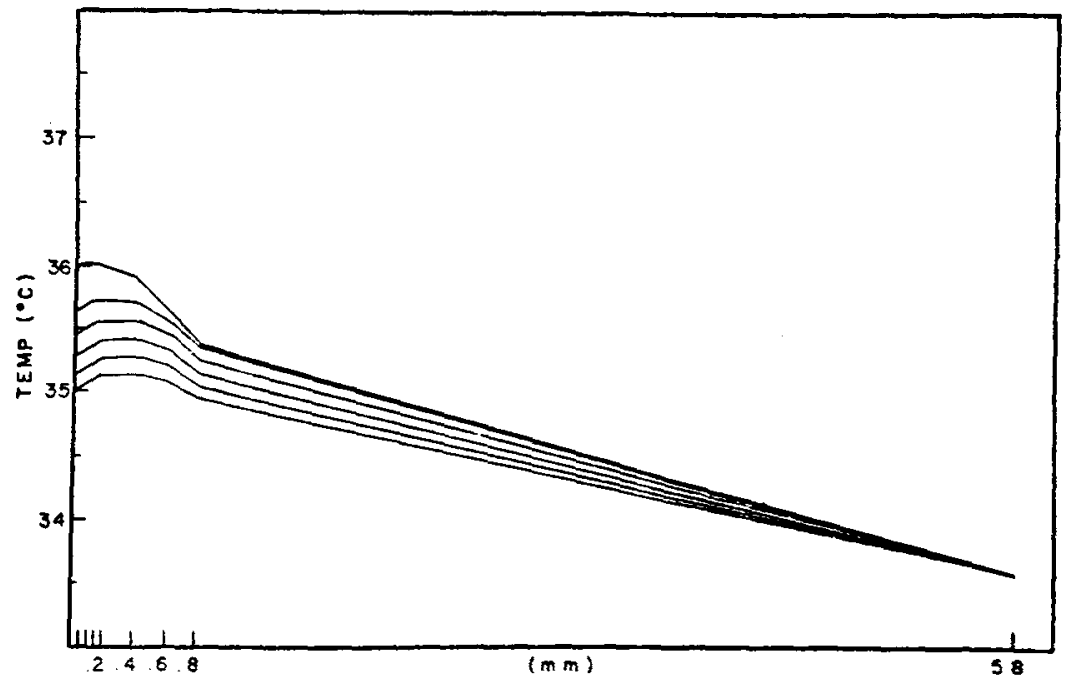




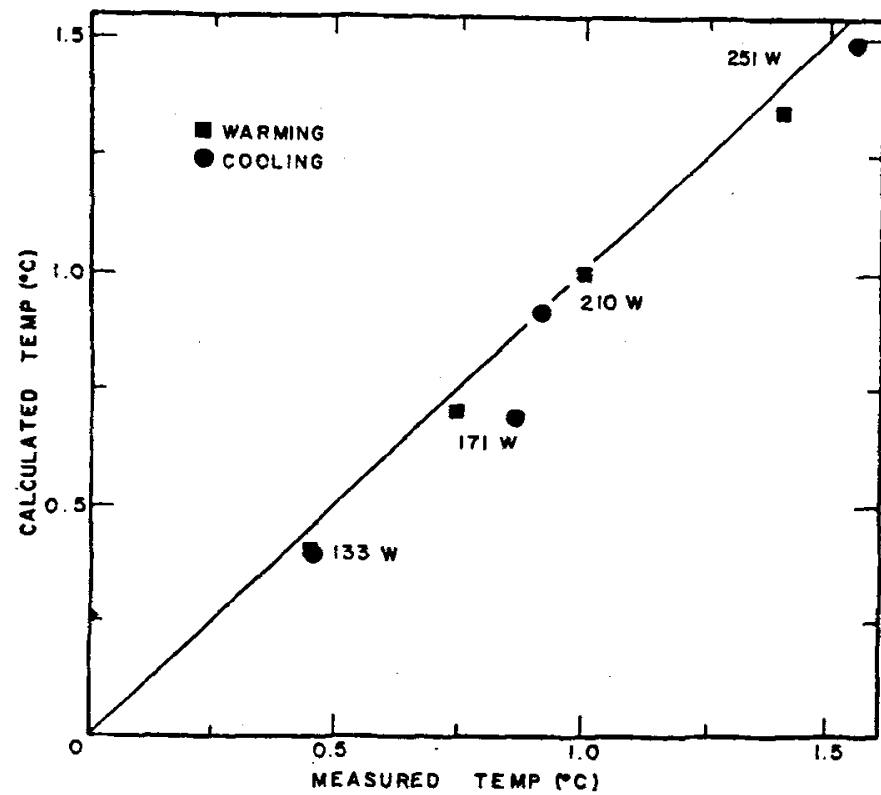

Fig. 14. Comparison of simulated and experimental measures of skin temperatures for warming and cooling at various reinforcement intensities.

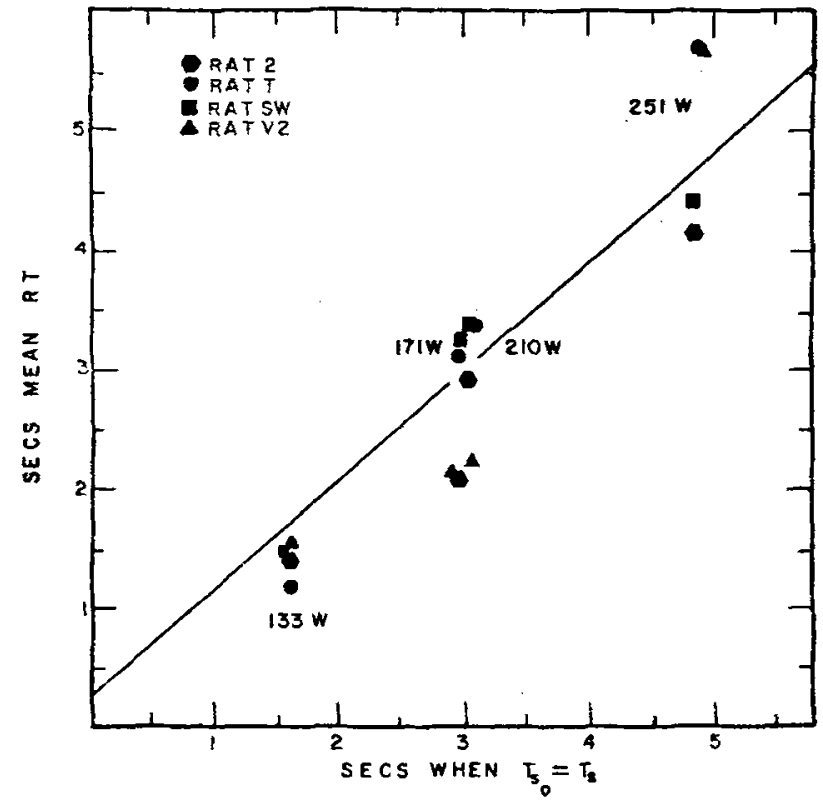

Fig. 15. Relationship between mean RT and time when (cool receptor layer of) skin cools to temperature that existed just prior to the response, for various reinforcement intensities.

seconds) for the skin temperature at $0.17 \mathrm{~mm}$ depth $\left(\mathrm{T}_{\mathrm{s}}\right)$ to return to the level that existed just prior to the onset of the reinforcement $\left(T_{\mathbf{s}_{0}}\right)$ is presented in Fig. 15.

The data for each animal at each reinforcement magnitude are presented, as well as the arithmetic means for all animals at each reinforcement intensity. The straight line is fitted by the method of least squares at each intensity. If the reaction time depended simply on the time the skin required to cool to the temperature that existed just prior to stimulation, the plot in Fig. 15 should be a linear relationship and the slope would be unity. The regression line in Fig. 15 has a slope of 0.88 and a $\mathrm{Y}$-axis intercept of 0.31 . Thus, we conclude that the parameter of the exponential probability density function, which describes the distributed reaction-time data of our experiment, is governed by the behavior of the receptor structure (the skin), $T_{s_{o}}-T_{s}$ in the Corbit (1970) model, and that the relevant cue for responding in our thermoregulation environment is cooling of the skin.

\section{REFERENCES}

Bazett, H. C. Temperature sense in man. In Anonymous (Ed.), Temperature, its measurement and control in science and industry. New York: Reinhard, 1941. Pp. 489-501.

Brearly, E. A., \& Kenshalo, D. R. Behavioral measurements of the sensitivity of cat's upper lip to warm and cool stimuli. Journal of Comparative \& Physiological Psychology, 1970, 70, 1.4 .

Bowman, K. Elektrophysiologische Untersuchungen über die Thermoreceptoren der Gesichtshaut. Acta Physiologica Scandinavica, 1958, 44, 79.

Carlisle, H. J. Heat intake and hypothalamic temperature during behavioral temperature regulation. Journal of Comparative \& Physiological Psychology, 1966, 61, 388-397

Carlisle, H. J. Initiation of behavioral responding for heat in a cold environment. Physiology \& Behavior, 1968, 3, 827-830.

Carlisle, $H$. J. Thermal reinforcement and temperature regulation. In W. C. Stebbins (Ed.), Animal psychophysics. New York: Appleton-Century-Crofts, 1970. Pp. 211-230.

Corbit, J. D. Behavioral regulation of body temperature. In J. D. Hardy, A. P. Gagge, and J. A. J. Stolwijk (Eds.), Physiological and behavioral temperature regulation. Springfield: Thomas, 1970. Pp. 777-801.

Dodt, E., \& Zotterman, Y. Mode of action of warm receptors. Acta Physiologica Scandinavica, 1952a, 26, 345-357.

Dodt, E., \& Zotterman, Y. The discharge of specific cold fibres at high temperatures (the paradoxical cold). Acta Physiologica Scandinavica, $1952 \mathrm{~b}, 36,358$.

Hensel, H. Physiologie der Thermoreception. Ergebnisse der Physiologie, 1952, 47, 166-368.

Hensel, H. Wärneempfindliches Rezeptorfeld in der Nasenregion der Katze. Naturwissenschaften, 1968a, 55, 233.

Hensel, H. Spezifische Wärmeimpulse aus der Nasenregion der Katze. Pflügers Archiv für die gesamte Physiologie des Menschen und der Tier, $1968 \mathrm{~b}, 302,374-376$.

Hensel, $H$. Temperature receptors in the skin. In J. D. Hardy, A. P. Gagge, and J. A. J. Stolwijk (Eds.), Physiological and behavioral temperature regulation. Springfield: Thomas, 1970, 442-453.

Hensel, H., Iggo, A., \& Witt, I. A quantitative study of sensitive cutaneous thermoreceptors with $\mathrm{C}$ afferent fibres. Journal of Physiology (London), 1960, 153, 113-126.

Hensel, H., \& Zotterman, Y. Quantitative Beziehungen zwischen der Entladung einzelner Kaltefasern und der Temperatur. Acta Physiologica Scandinavica, 1951, 23, 291-319.

Iggo, A. Cutaneous thermoreceptors in primates and sub-primates. Journal of Physiology, 1969, 200, 403-430.

Iriuchijima, J., \& Zotterman, Y. The specificity of afferent cutaneous $C$ fibers in mammals. Acta Physiologica Scandinavica, 1960, 49, 267-278.

Kenshalo, D. R. The temperature sensitivity of the furred skin of cats. Journal of Physiology, 1964, 172, 439.

Kenshalo, D. R. Behavioral and electrophysiological responses of cats to thermal stimuli. In D. R. Kenshalo (Ed.), The skin senses. Springfield: Thomas, 1968. Pp. 400-422. 
Kenshalo, D. R. Cutaneous temperature receptors-Some operating characteristics for a model. In J. D. Hardy, A. P. Gagge, and J. A. J. Stolwijk (Eds.), Physiological and behavioral temperature regulation. Springfield: Thomas, 1970. Pp. 802-818.

Kenshalo, D. R. Psychophysical studies of temperature sensitivity. In W. D. Neff (Ed.), Contributions to sensory physiology. Vol. 4. New York: Academic Press, 1970. Pp. 19-20.

Kenshalo, D. R., \& Brearly, E. A. Electrophysiological measurements of the sensitivity of cat's upper lip to warm and cool stimuli. Journal of Comparative \& Physiological Psychology, 1970, 70, 5-14.

Kenshalo, D. R., Duncan, D. G., \& Weymarck, C. Thresholds for thermal stimulation of the inner thigh, footpad, and face of cats. Journal of Comparative \& Physiological Psychology, $1967,63,133$.

Luce, R. D., \& Green, D. M. Detection of auditory signals presented at random times, II. Perception \& Psychophysics, $1970,7,1-14$

Luce, R. D., \& Green, D. M. A neural timing theory for response times and the psychophysics of intensity. Psychological Review, 1972, 79, 14-57.
McGill, W. J. Stochastic latency mechanisms. In R. D. Luce, R. R. Bush, and E. Galanter (Eds.), Handbook of mathematical psychology. Vol. 1. New York: Wiley, 1963. Pp. 309-360.

Stolwijk, J. A. J., \& Hardy, J. D. Skin and subcutaneous temperature changes during exposure to intense thermal radiation. Journal of Applied Physiology, 1965, 20, 1006-1013.

Stolwijk, J. A. J., \& Wexler, I. Peripheral nerve activity in response to heating the cat's skin. Journal of Physiology, $1971,214,377-392$.

Weiss, B., \& Laties, V. G. Behavioral thermoregulation. Science, $1961,133,1338-1344$.

Zotterman, Y. Specific action potentials in the lingual nerve of the cat. Scandinavica Archiv für Physiologie, 1936, 75, 105-119.

(Received for publication A pril 26, 1973; revision received June $24,1973$. 